\title{
Medium-velocity superconducting cavity for high accelerating gradient continuous-wave hadron linear accelerators
}

\author{
K. McGee $\odot,{ }^{1}$ S. Kim, ${ }^{1}$ K. Elliott $\odot,{ }^{1}$ A. Ganshyn $\odot,{ }^{1}$ W. Hartung,,${ }^{1}$ E. Metzgar, ${ }^{1}$ P. Ostroumov, ${ }^{1}$ \\ L. Popielarski, ${ }^{1}$ J. Popielarski, ${ }^{1}$ A. Taylor, ${ }^{1}$ T. Xu, ${ }^{1}$ M. P. Kelly, ${ }^{2}$ B. Guilfoyle, ${ }^{2}$ and T. Reid ${ }^{2}$ \\ ${ }^{1}$ Michigan State University, East Lansing, Michigan 48824, USA \\ ${ }^{2}$ Argonne National Laboratory, Argonne, Illinois 60439, USA
}

(Received 29 January 2021; accepted 4 October 2021; published 18 November 2021)

\begin{abstract}
We present the first rf studies of the medium- $\beta$ superconducting radio frequency (SRF) elliptical cavities designed for Michigan State University's Facility for Rare Isotope Beams (FRIB) energy upgrade linac. The proposed energy upgrade for this continuous-wave $(\mathrm{CW})$ superconducting linac will double the final beam energy from 200 to $400 \mathrm{MeV} / \mathrm{u}$ for the heaviest uranium ions within the $80 \mathrm{~m}$ of space available in the FRIB tunnel. Two prototype $\beta_{\text {opt }}=0.65644 \mathrm{MHz}$ five-cell elliptical SRF cavities were fabricated and tested to validate the novel cavity design with three conventional rf recipes: (1) Electropolishing (EP-only), (2) $\mathrm{EP}+48 \mathrm{~h} 120^{\circ} \mathrm{C}$ bake (EP + baking), and (3) Buffered chemical Polishing $+48 \mathrm{~h} 120^{\circ} \mathrm{C}$ bake (BCP + baking). The EP-only recipe achieved a $2 \mathrm{~K}$ quality factor $\left(Q_{0}\right)$ of $2.3 \times 10^{10}$ at the FRIB energy upgrade design accelerating gradient $\left(E_{\text {acc }}\right)$ of $17.5 \mathrm{MV} / \mathrm{m}$, and $Q_{0}$ of $1.2 \times 10^{10}$ at a maximum gradient of $26 \mathrm{MV} / \mathrm{m}$, where the gradient was ultimately limited by the available rf amplifier power available for this test. These results validate the potential of the novel $644 \mathrm{MHz}$ medium- $\beta$ cavity design and motivate its use in future high- $\mathrm{Q}_{0}$ development work. The multipacting band, which exists at $E_{\text {acc }} \approx 10 \mathrm{MV} / \mathrm{m}$, was completely conditioned in the continuous-wave mode. We also observed that combining the $48 \mathrm{~h} 120^{\circ} \mathrm{C}$ baking treatment with EP did not improve the EP-only cavity performance at $2 \mathrm{~K}$ due to increased residual resistance $\left(R_{0}\right)$ and increased medium-field Q-slope. $\mathrm{BCP}+$ baking was also found to produce lower $\mathrm{Q}_{0}$ in this cavity due to increased medium-field Q-slope. The mechanical modes of this cavity were measured at room temperature, verifying that the quality factor of the dominant "accordion" mechanical mode these medium- $\beta$ cavities are particularly vulnerable to is close to that of the $1.3 \mathrm{GHz}$ TESLA cavities. Thus, this mode arising from the novel geometry of these cavities is shown to not be of excessive concern for resonance control.
\end{abstract}

DOI: 10.1103/PhysRevAccelBeams.24.112003

\section{INTRODUCTION}

To meet the FRIB energy upgrade project (FRIB400 [1]) design specifications, multiple cavity designs and operating frequencies were investigated. With all cavity options scaled to a peak electric field of $40 \mathrm{MV} / \mathrm{m}$, it was determined that a novel five-cell elliptical $644 \mathrm{MHz}$ cavity with $\beta_{\text {opt }}=0.65$ was the only one able to achieve $400 \mathrm{MeV} / \mathrm{u}$ for uranium ions within the $80 \mathrm{~m}$ available in the FRIB linac tunnel [2]. This design minimized numbers of additional cavities and cryomodules needed, provided the cavities can be processed to achieve $E_{\text {acc }}$ of $17.5 \mathrm{MV} / \mathrm{m}$ with a $\mathrm{Q}_{0}$ of at least $2 \times 10^{10}$. This cavity design also achieves the lowest total dynamic heat load in

Published by the American Physical Society under the terms of the Creative Commons Attribution 4.0 International license. Further distribution of this work must maintain attribution to the author(s) and the published article's title, journal citation, and DOI. the upgraded linac segment [2]. A summary of fundamental parameters for this cavity is presented in Table I.

With the design established, the goal of this work is to validate that the novel FRIB400 cavity has good potential to serve as a testbed for high-Q recipe development. We thus seek to demonstrate that reasonable rf performance can be achieved with at least one of the number of conventional rf surface processing recipes. Technologies for processing superconducting radio frequency (SRF) cavities have matured over a wide range of operating frequencies and velocities. With multiple applications and the inertia provided by a long history in the literature, the $1.3 \mathrm{GHz}$ TESLA cavities have persisted as the testbed of choice for novel high-gradient and high-Q cavity processing treatments [3]. In these cavities, electropolishing (EP) [4] evolved into the "standard ILC" treatment developed for the International Linear Collider (ILC) [5]. In the context of mass-production for the European X-ray Free Electron Laser, the ILC recipe was shown to be highly repeatable on over $8001.3 \mathrm{GHz}$ SRF cavities, with the average cavity 
TABLE I. Cavity specifications.

\begin{tabular}{lcc}
\hline \hline Parameter & Value & Units \\
\hline Frequency & 644 & $\mathrm{MHz}$ \\
Geometrical $\beta$ & 0.61 & \\
Optimal $\beta$ & 0.65 & \\
Aperture diameter & 83 & $\mathrm{~mm}$ \\
Effective length $L_{\text {eff }}$ & 71.0 & $\mathrm{~cm}$ \\
Number of cells & 5 & \\
Geometric shunt impedance $R / Q$ & 368 & $\Omega$ \\
Geometry factor $G$ & 188 & $\Omega$ \\
$E_{\text {peak }}$ & 40 & $\mathrm{MV} / \mathrm{m}$ \\
$B_{\text {peak }}$ & 77.5 & $\mathrm{mT}$ \\
Accelerated gradient $E_{\text {acc }}$ & 17.5 & $\mathrm{MV} / \mathrm{m}$ \\
$E_{\text {peak }} / E_{\text {acc }}$ & 2.28 & \\
$B_{\text {peak }} / E_{\text {acc }}$ & 4.42 & $\mathrm{mT} /(\mathrm{MV} / \mathrm{m})$ \\
\hline \hline
\end{tabular}

gradient exceeding specifications by $25 \%$ [6]. Initial explorations of nitrogen-doping (N-doping) [7] and nitrogen-infusion [8] for high-Q applications are also rooted in TESLA cells, and have seen early success and rapid industrialization for the Linac Coherent Light Source-II (LCLS-II) high- $\beta 1.3 \mathrm{GHz}$ cavities with improved $\mathrm{Q}$ at $2 \mathrm{~K}$ $[9,10]$. Low-velocity $\left(\beta_{\text {opt }}=\mathrm{v} / \mathrm{c}<0.5\right)$ cavity designs and surface treatments have also been well optimized. The EPtreated performance of low- $\beta$ coaxial cavities is approaching state-of-the-art performance of the TESLA cavities processed with the standard ILC recipe [11]. In addition to EP, buffered chemical polishing (BCP) has also seen success in low- $\beta$ cavities: in the recent baseline production cycle of approximately $300 \beta_{\text {opt }}=0.04$ to 0.53 quarterwave and half-wave resonators for FRIB, BCP consistently met or exceeded all facility specifications [12,13].

Previous $\mathrm{Q}_{0}$ development in this medium- $\beta$ operating regime aimed at $\mathrm{Q}_{0}$ requirements around an order of magnitude lower than the FRIB400 minimum requirements. The Spallation Neutron Source (SNS) specifications call for $\mathrm{Q}_{0} \geq 5 \times 10^{9}$ at $E_{\text {acc }}=10.1 \mathrm{MV} / \mathrm{m}$ for $\beta=0.61$ cavities, and the European Spallation Source (ESS) specifications call for $\mathrm{Q}_{0}>5 \times 10^{9}$ at $E_{\text {acc }}=16.7 \mathrm{MV} / \mathrm{m}$ for $\beta=0.67$ cavities. SNS and ESS both applied BCP treatments and achieved their respective minimum requirements. While achieving their specifications, the SNS and ESS cavities did not consistently achieve $\mathrm{Q}_{0}>2 \times 10^{10}$ $[14,15,16]$ required for the FRIB400 upgrade.

Furthermore, the size of the FRIB upgrade cavity presents technical challenges for BCP, given the need for effective cavity surface cooling, and adequate flow rate to avoid the formation of nitrogen dioxide bubbles on the cavity surfaces during BCP [17]. Moreover, BCP yields a surface roughness nearly an order of magnitude higher than EP, where differently orientated crystals experience preferential etching $[17,18]$, which can make reaching higher gradients without field emission (FE) more challenging.
Validating the EP-only performance of this cavity is of principal interest for this work as this treatment serves as the baseline for $\mathrm{N}$-doping. Acceptable EP performance will justify pursuit of $\mathrm{N}$-doping development in this medium- $\beta$ geometry. Technical challenges inherent to applying EP to medium- $\beta$ cavities [19] will be discussed later. The standard ILC EP treatment, augmented with low-temperature baking to remove high-field Q-slope [20], has been extensively developed in $1.3 \mathrm{GHz}$ high- $\beta$ cavities to achieve high $\mathrm{Q}$ and high gradient. EP also showed good high-Q and high-gradient performance in lower-frequency high- $\beta$ cavities such as SNS high- $\beta(\beta=0.81,805 \mathrm{MHz})$ and Proton Improvement Plan II (PIP-II) high- $\beta$ ( $\beta=0.92$, $650 \mathrm{MHz}$ ) cavities [21,22,23]. However, it is not obvious how the $644 \mathrm{MHz}$ FRIB prototype cavities will respond to the ILC EP process.

Specifically, cavity performance improves with the reduction of the rf surface resistance $\left(R_{\mathrm{S}}\right)$, which has temperature-dependent and temperature-independent parts (1) [24],

$$
R_{\mathrm{s}}=R_{\mathrm{BCS}}(T)+R_{0},
$$

$R_{\mathrm{BCS}}$, which is derived from the Bardeen-Cooper-Schrieffer theory of superconductivity, has key dependencies (2) on the operating frequency $(\omega)$, the superconducting energy gap $(\Delta)$, and the material parameter (A) [24],

$$
R_{\mathrm{s}}=\frac{A \omega^{2}}{T} e^{-\frac{\Delta}{k_{B} T}}+R_{0}
$$

The residual resistance $\left(R_{0}\right)$ depends on various factors such as flux trapping, surface defects, and impurity content. Different cavity surface treatments and different operating conditions such as frequency, temperature, and accelerating gradient, will affect the surface resistance. Moreover, the electrodynamic and physical properties of the larger, lowerfrequency medium- $\beta$ cavity make it difficult to predict how $R_{\mathrm{S}}$ will respond to different surface processing techniques.

Thus, with the two prototype cavities available to FRIB for testing, S65-001 and S65-002, we elected to begin with a trial of ILC-like EP with low-temperature baking in S65-001, and BCP with low-temperature baking in cavity S65-002. Anticipating eventual interest in $\mathrm{N}$-doping these cavities, we then chose to reset S65-001 and test EP-only (no baking) to baseline future $\mathrm{N}$-doped S65-001 performance.

\section{SURFACE PROCESSING AND CAVITY PERFORMANCE}

The initial surface treatment of S65-001 was based on the standard ILC recipe for EP with a modified hydrogen degassing step. The $150 \mu \mathrm{m}$ bulk EP at Argonne National Laboratory (ANL) with the modified electrode resulted in an average of $199 \mu \mathrm{m}$ of removal from the beam pipes 
and an average of $149 \mu \mathrm{m}$ from the equatorial regions. The cavity was then baked for the purpose of hydrogen degassing: first at $350^{\circ} \mathrm{C}$ for $12 \mathrm{~h}$, then at $600^{\circ} \mathrm{C}$ for $10 \mathrm{~h}$. The cavity was then mechanically tuned to $97 \%$ cellto-cell field flatness and shipped back to ANL for the final $20 \mu \mathrm{m} \mathrm{EP}$, high-pressure water rinsing and clean assembly. For the ensuing EP + baking test and after the final clean assembly, S65-001 was baked at $120^{\circ} \mathrm{C}$ for $48 \mathrm{~h}$ in situ, i.e., while assembled to the dunk-test insert and under vacuum. For the EP-only test, S65-001 was then "reset" using $40 \mu \mathrm{m}$ EP at ANL, with the last $10 \mu \mathrm{m}$ being "cold-EP" performed at $15-17{ }^{\circ} \mathrm{C}$. High-pressure water rinsing (HPR) and clean assembly for this cavity was then conducted in FRIB cleanroom facilities.

Since test results of medium- $\beta$ multicell cavities processed by EP have not been reported before, the process is worth describing, and in particular, the EP cathode geometry. The generic concern about the EP in the medium- $\beta$ cavities is relatively high aspect ratio of the equator radius $197 \mathrm{~mm}$ to the iris-to-iris distance (single-cell length) $142 \mathrm{~mm}$, compared to the other high- $\beta$ cavities [2]. The greater distance of the cavity equator to the cathode, in general, effectively increases the electrolyte resistance, decreasing the cavity surface current as a function of position along the beam axis, which results in nonuniform surface removal [19]. To reduce this effect, the cathode at the position of the beam pipes and irises was masked with Teflon tape as shown in Fig. 1(a). Additional attachments, so-called doughnuts as shown in Fig. 1(b), were assembled onto the cathode at the position of the equators. The surface removal at the locations as shown in Fig. 1(a) was measured and the results are as shown in Fig. 1(c). The average surface removal at the equators is $75 \%$ of the average surface removal at the beam pipes. This ratio is reasonably high compared to not only medium- $\beta$ single cell cavities but also TESLA cavities. However, the authors would like to note that it is possible that the surface removal around the irises, which was not accessible for the ultrasonic thickness measurements due to the stiffening rings, was higher than the beam pipes due to closer proximity to the unmasked aluminum doughnuts.

S65-002 underwent BCP treatment in FRIB baseline production $\mathrm{BCP}$ facilities mounted vertically and without additional cooling beyond that provided by the acid itself [10]. The treatment cycle consisted of a $120 \mu \mathrm{m}$ etch, $600^{\circ} \mathrm{C}$ baking, another $20 \mu \mathrm{m} \mathrm{BCP}$ etch, and an in situ $120^{\circ} \mathrm{C}$ bake for $48 \mathrm{~h}$ (BCP + baking).

The $2 \mathrm{~K}$ Q-curves for the two S65-001 tests, EP-only and EP + baking, are presented in Fig. 2(a). The EP-only treatment achieved $\mathrm{Q}_{0}$ of $2.3 \times 10^{10}$ at $E_{\text {acc }}=17.5 \mathrm{MV} / \mathrm{m}$, exceeding the FRIB upgrade requirements. The maximum $E_{\text {acc }}$ was $26.1 \mathrm{MV} / \mathrm{m}$, limited by the available amplifier power in the test. The EP + baking treatment achieved $\mathrm{Q}_{0}$ of $1.9 \times 10^{10}$ at maximum gradient $E_{\text {acc }}=17.5 \mathrm{MV} / \mathrm{m}$. This maximum gradient was limited in amplifier power by

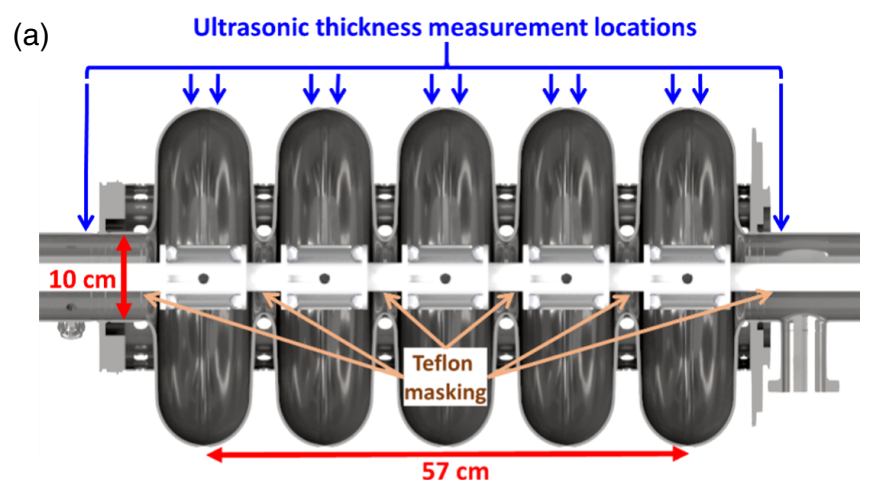

(b)

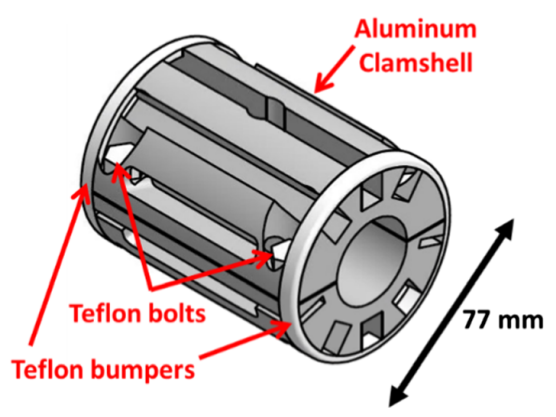

(c)

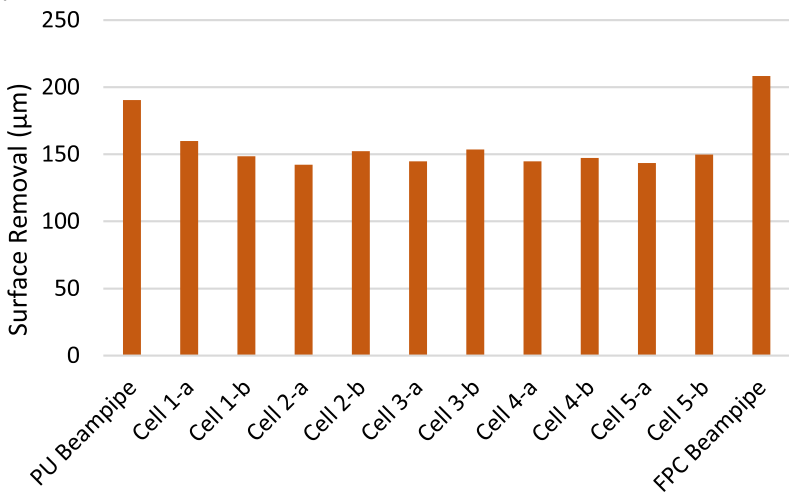

FIG. 1. S65-001 surface removal with bulk EP. (a) EP cathode integrated with the cavity. This also shows the doughnuts on the cathode at the equator locations and Teflon shielding at the iris locations. Red arrows indicate the locations for ultrasonic thickness measurements. (b) "Doughnut" to increase the cathode radius at the equator locations. Five doughnuts are attached to a cylindrical aluminum tube with $34 \mathrm{~mm}$ diameter; the remaining area of the aluminum tube is masked by Teflon tape. (c) Surface removal at the locations of the beampipes and equators. Since the outer surfaces of the irises were not easy to access due to stiffening rings, we measured the beampipes as a representation of the surfaces at a small radius. PU and FPC denote the pickup and fundamental power coupler, respectively.

unconditionable field emission. The measured field emission (FE) had negligible effect on $\mathrm{Q}_{0}$, since it remained well below $100 \mathrm{mR} / \mathrm{h}$ at all fields. It has been found that $\mathrm{FE}$ must exceed $100 \mathrm{mR} / \mathrm{h}$ before causing measurable Q-drop [25]. Furthermore, the $\mathrm{Q}_{0}$ observed at fields below $10 \mathrm{MV} / \mathrm{m}$, a field level where FE was absent in all cavities tested, was the same between field-emitting and 


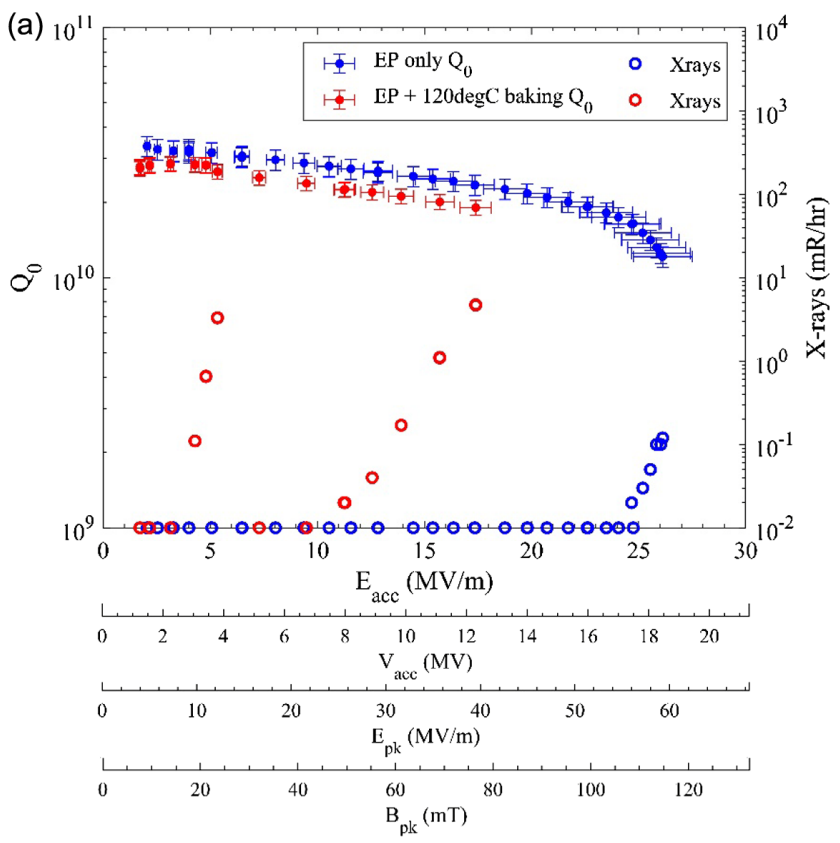

(b)

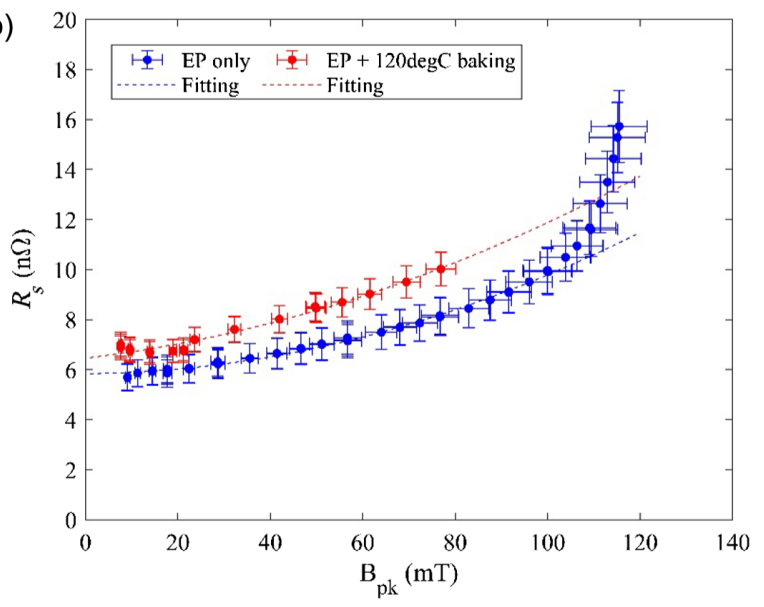

FIG. 2. Results of the high-power rf testing of $644 \mathrm{MHz} \beta_{\mathrm{opt}}=$ 0.65 cavities at MSU/FRIB. (a) $2 \mathrm{~K} Q$ curves comparing EP only vs EP $+120^{\circ} \mathrm{C}$ baking recipes in S65-001. The blue circles are the EP-only case and the red circles are the EP $+120^{\circ} \mathrm{C}$ baking case. The open circles are field-emission $\mathrm{X}$ rays, as read on the right-hand vertical scale. In all trials FE remained well below $100 \mathrm{mR} / \mathrm{h}$, where noticeable degradation in Q due to FE ocurrs at $100 \mathrm{mR} / \mathrm{h}$ and above [25]. The color code is the same as for $\mathrm{Q}_{0}$. (b) The same results as in (a) but scaled to show the rf surface resistance $R_{\mathrm{s}}$. The dashed lines are second-degree polynomial fits to each data set. These fits were done for the data, for which $B_{\mathrm{pk}}$ is in the range of 20 to $100 \mathrm{mT}$, but they are extrapolated into the range of 1 to $120 \mathrm{mT}$ for readability.

nonfield-emitting cavities. This suggests there was no inherent $\mathrm{Q}_{0}$ degradation as a result of the presence of field-emitting contaminants. Given this, and the fact that the measured FE was below $100 \mathrm{mR} / \mathrm{h}$, it was concluded that the presence of FE affected the maximum achievable gradient only, and had a negligible effect on measured $\mathrm{Q}_{0}$. Further details regarding the relationship between $\mathrm{Q}_{0}$ and field emission are outside of the scope of this paper, and are thus not discussed.

The authors speculate that the contaminents resulting in this level of field emission originated in the HPR system, as presented in Sec. IV. Due to the limited spanning angle of the robotic arm, the HPR nozzle needed to have at least twelve holes to cover the entire cavity inner surface. This increase in hole humber from the two- to three-hole nozzles decreased the velocity of the water jets and thus lowered the impact force of the water against the cavity walls. The in situ baking of the cavity occurred after clean assembly, and thus is unlikely to have been the direct cause of the field emission.

In Fig. 2(b), these same results are presented in terms of the rf surface resistance $\left(R_{\mathrm{S}}\right)$ vs the peak magnetic field, $B_{\mathrm{pk}}$, which more clearly shows the nature of the difference in $R_{\mathrm{S}}$ introduced by baking in EP-ed cavities. The baked cavity's $R_{\mathrm{S}}$ is increased above that of the unbaked cavity both by a slightly steeper slope in $R_{\mathrm{s}} \mathrm{vs} B_{\mathrm{pk}}$, and an overall upwards shift, seen at low fields. As observed in Ref. [26], both linear and quadratic dependencies exist between $R_{\mathrm{S}}$ and field strength.

In general, the medium-field Q-slope is not solely affected by the intrinsic properties of the rf surface treatment. Extrinsic factors, such as localized defects or cavity contaminants also contribute. However, since this study encompasses the effect of baking on the FRIB400 cavity performance, we still find it useful to quantify the change in the Q-slope after the $120^{\circ} \mathrm{C}$ in situ baking treatment. While all physical factors governing the medium- and high-field Q-slopes are not totally captured by a simple polynomial fit, it is instructive to compare our results to those presented in Ref. [26], which employed a second-order polynomial fit. This fitting further can shed light on what types of sources caused the additional losses after baking. Namely, if the losses originated from contaminations that caused excessive Joule heating or dielectric losses, we would be able to see an increase in the second-order term, regardless of how the contaminants are localized.

To aid to benchmarking the novel cavity's performance, we fit the data in Fig. 2(b) to the second-order polynomial equation:

$$
R_{\mathrm{s}}[\mathrm{n} \Omega]=C_{0}+C_{1} b+C_{2} b^{2}
$$

where $b$ is normalized to $B_{\mathrm{pk}}$ defined by $b=B_{\mathrm{pk}} / B_{\mathrm{pk}, 0}$, where $B_{\mathrm{pk}, 0}$ is the $B_{\mathrm{pk}}$ at the design accelerating gradient, $77.5 \mathrm{mT}$. $C_{0}, C_{1}$, and $C_{2}$ are respectively the zeroth-, first-, and second-order coefficients, which represent the relative strengths of field-independent, linear, and quadratic components of $R_{\mathrm{S}}$ at the design gradient. The fitting results are as shown in Table II. These results indicate that the zerothand first-order terms clearly contribute to the baked cavity's higher $R_{\mathrm{s}}$ at the operating gradient $(b=1)$. 
TABLE II. Second-order polynomial fitting results of the fielddependent $R_{\mathrm{s}}$.

\begin{tabular}{lccc}
\hline \hline Case & $C_{0}$ & $C_{1}$ & $C_{2}$ \\
\hline EP-only & $5.8 \pm 0.023$ & $0.15 \pm 0.077$ & $2.3 \pm 0.052$ \\
EP + baking & $6.5 \pm 0.042$ & $1.7 \pm 0.19$ & $1.9 \pm 0.18$ \\
\hline \hline
\end{tabular}

Figure 3 offers insight into the zeroth- and first-order terms. In plotting $R_{\mathrm{s}} \mathrm{vs} T_{c} / T$, the $\mathrm{BCS}$ resistance, $R_{\mathrm{BCS}}$, is identified as the temperature-dependent decrease of $R_{\mathrm{s}}$, and the residual resistance $\left(R_{0}\right)$ is roughly identified with the temperature-independent (flat-line limit) of $R_{\mathrm{S}}$ at low $T$. The zeroth-order term contains the sum of the fieldindependent $R_{0}$ and the temperature-dependent $R_{\mathrm{BCS}}$, however, at low temperatures (e.g., less than $2 \mathrm{~K}$ ) the overwhelming contribution to this term is $R_{0}$, and thus the zeroth-order term is largely taken to correspond to $R_{0}$, which is greater in the baked cavity (4). Near the right-hand edge of the plot in Fig. 3, we see that $R_{0}$ is not only greater in the baked cavity, but also the dominant contributor to $R_{\mathrm{S}}$ around $2 \mathrm{~K}$, the FRIB operating temperature. Thus, even though baking visibly reduced $R_{\mathrm{BCS}}$, this did not lead to better cavity performance at $2 \mathrm{~K}$.

The first-order term, associated with the medium-field Q-slope (MFQS), can result from hysteresis losses due to Josephson fluxons formed at oxide-filled boundaries

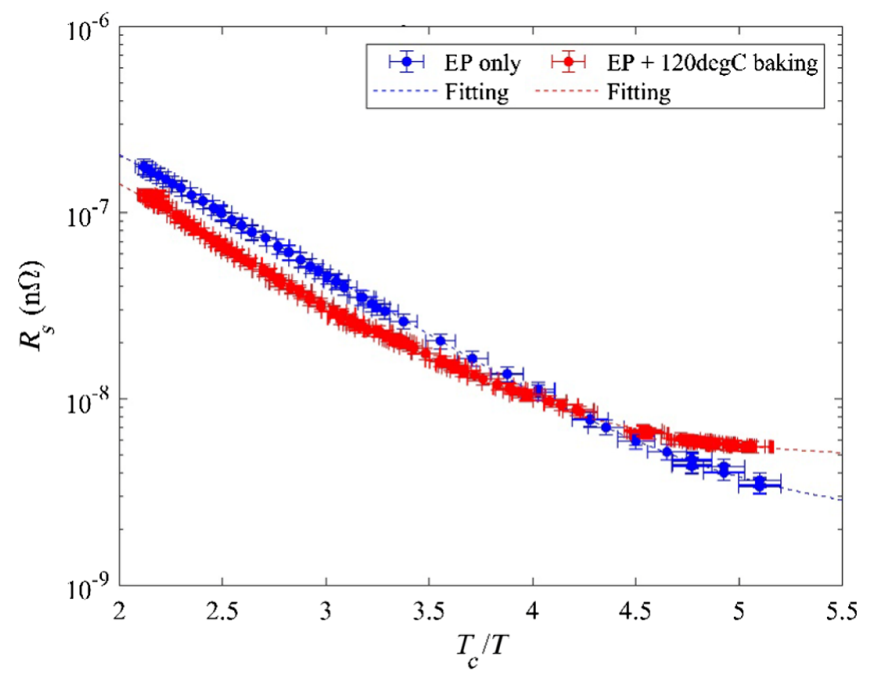

FIG. 3. $R_{\mathrm{s}}$ as a function of $T_{\mathrm{c}} / T$, where $T_{\mathrm{c}}=9.2 \mathrm{~K}$, the critical temperature of niobium. $T$ is taken as the liquid helium bath temperature. These data were recorded at constant $E_{\text {acc }}$ of $2 \mathrm{MV} / \mathrm{m}$ for the EP-only case, and at approximately $4 \mathrm{MV} / \mathrm{m}$ for the $\mathrm{EP}+120^{\circ} \mathrm{C}$ baking case. This difference in field level is not regarded as significant because the total surface resistance, $R_{\mathrm{s}}$, is relatively field independent at these low fields. Both datasets are fitted to Eq. (2), $R_{\mathrm{S}}=\frac{A \omega^{2}}{T} e^{-\frac{\Delta}{K_{B} T}}+R_{0}$, where the fitted parameters are (1) EP-only: $A=2.6 \times 10^{-24}, \frac{\Delta}{k_{B} T_{c}}=1.9$, $R_{0}=2.2 \mathrm{n} \Omega$, and (2) EP + baking: $A=2.1 \times 10^{-24}, \frac{\Delta}{k_{B} T_{c}}=2.0$, $R_{0}=4.8 \mathrm{n} \Omega$. penetrating within the niobium, along microcracks or grain boundaries [26,27]. Less intrinsic contributions also come from defects causing localized heating [26]. Baking is known to increase the first-order term by as much as a factor of 2 in $1.47 \mathrm{GHz}$ elliptical cavities [26,28]. We also see a large increase in this term after baking the FRIB $644 \mathrm{MHz}$ cavity.

Not all contributions to the second-order term are known, and it is not the aim of this paper to investigate these. However, we note that possible contributors to the secondorder term are the thermal feedback effect and the nonlinear pair breaking effect $[26,28]$. We did not observe that baking caused significant change in this term, which would further indicate that there was no significant increase in sources of Joule heating or dielectric losses after the baking treatment. With regard to the thermal-feedback effect, we note that the outer surface of the cavity was held in the same thermal environment in each test to mitigate any possible differences in the thermal feedback effect between tests. Recall the EP-only test was conducted after the EP + baking test, after a $40 \mu \mathrm{m}$ EP reset of the cavity (S65-001).

In the EP-only case, second-order fitting becomes invalid after $B_{\mathrm{pk}}$ increases beyond $100 \mathrm{mT}$, once the high-field Q-slope becomes evident. In situ $120^{\circ} \mathrm{C}$ baking is known to reduce high-field Q-slope in ILC TESLA cavities, however in the case of the FRIB $644 \mathrm{MHz}$ cavities operating at a gradient of $17.5 \mathrm{MV} / \mathrm{m}$, baking to eliminate the high-field Q slope would not lead to a decrease in $R_{\mathrm{s}}$ until the cavity exceeds a $B_{\mathrm{pk}}$ of $110 \mathrm{mT}, \sim 40 \%$ higher than its design gradient.

It is noteworthy that the effect of $120^{\circ} \mathrm{C}$ baking in the FRIB $644 \mathrm{MHz}$ cavity differs from the effect in $1.3 \mathrm{GHz}$ ILC TESLA cavities, where it is part of typical highgradient treatment $[19,29,30]$. At $644 \mathrm{MHz}$, and a different $B_{\mathrm{pk}}$ (an effect of the medium- $\beta$ geometry), the FRIB upgrade cavity sees a higher contribution of $R_{0}$ to $R_{\mathrm{S}}$ than in an ILC TESLA cavity. This is in part due to the fact that the fraction of $R_{0}$ due to the trapped magnetic flux scales approximately with the square root of the operating frequency, whereas the $R_{\mathrm{BCS}}$ scales with the square of the operating frequency [31]. It is further noteworthy that the operating gradient of the FRIB cavity is lower than the typical high-gradient cavity, such that it falls within the medium-field Q-slope region. As noted, previous studies, both theoretical and experimental, [26,28,32] find that baking increases the medium-field Q slope, and our results confirm this. Due to this consistency, we are unmotivated to pursue baking treatments further in this frequency and gradient regime.

The Argonne EP facilities used to treat the EP-only and the EP + Baking cavity are well-established and have shown good consistency in cavity performance curves $[33,34]$ over the last decade, giving the authors confidence in the statistical relevance of the results obtained from the FRIB EP cavity test. 


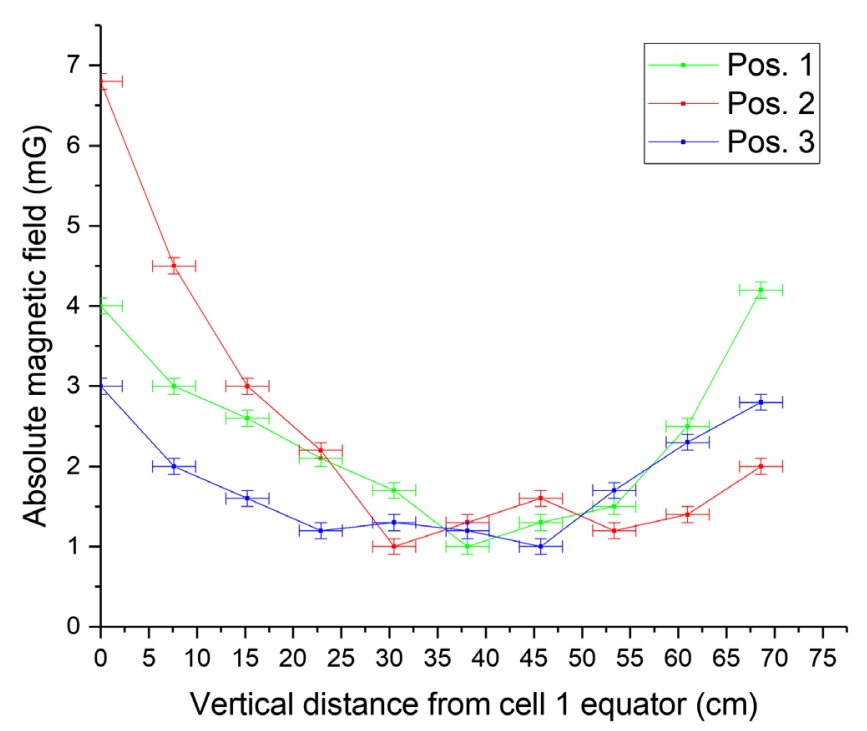

FIG. 4. Background magnetic field in the vertical test dewar. The fields were measured by lowering fluxgate magnetostatic sensors in increments to create a vertical scan at three different horizontal positions. The sensor locations were offset from the center axis by the same distance as the cavity equator, and they are spaced by $120^{\circ}$ in the azimuthal direction from each other. These were measured with the cavity installed in the dewar together with the dewar lid; however, this measurement could only be conducted at room temperature, not at $2 \mathrm{~K}$.

The background magnetic field was below $7 \mathrm{mG}$ for all locations in these tests, with an average of $2.23 \mathrm{mG}$ as shown in Fig. 4. Despite the low average background field, the first and fifth cells are subject to elevated, and potentially problematic, background fields given the sensitivity of these cavities to flux trapping. This will be especially true after planned $\mathrm{N}$-doping, and will be addressed using compensation coils in future tests to more uniformly minimize the background magnetic field.

The $2 \mathrm{~K}$ Q-curve of the $\mathrm{BCP}+$ baking treatment is shown in Fig. 5 juxtaposed with the EP + baking results. The $\mathrm{BCP}+$ baking cavity shows a relatively strong lowfield Q-slope, which appears as an inverse Q-slope at $B_{\mathrm{pk}}$ lower than approximately $20 \mathrm{mT}$. As a result, the BCP + baking cavity's $Q_{0}$ values below $60 \mathrm{mT}$ exceed the EP + baking $Q_{0}$ values at the same field levels. However, a stronger medium-field Q-slope is seen in the $\mathrm{BCP}+$ baking cavity, and its $Q_{0}$ values are clearly beginning to dip below those of the EP + baking cavity before reaching the FRIB upgrade operating gradient of $17.5 \mathrm{MV} / \mathrm{m}$. Field emission in the $\mathrm{BCP}+$ baking cavity prevented testing above $15.6 \mathrm{MV} / \mathrm{m}$ at $2 \mathrm{~K}$; however, this field emission was not the cause of the Q-drop observed in that cavity since x-ray degradation of $Q_{0}$ begins at an emission level above approximately $100 \mathrm{mR} / \mathrm{h}$, which is well above our measured x-ray emission level. We thus conclude that the measured $Q_{0}$ in this cavity up to $E_{\text {acc }}=14 \mathrm{MV} / \mathrm{m}$ or

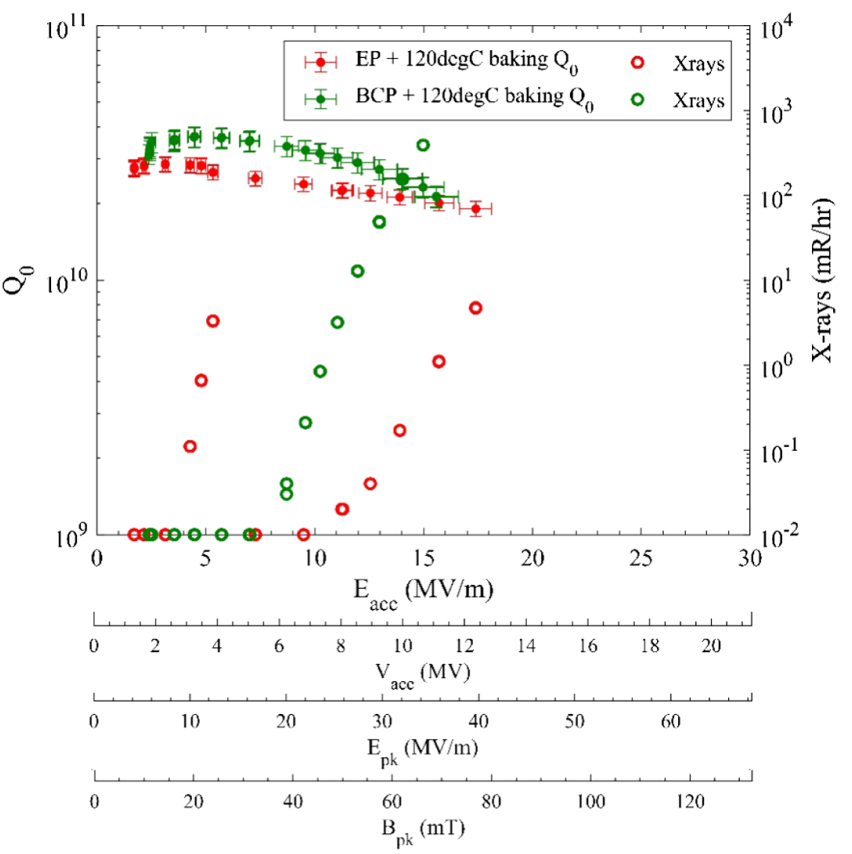

FIG. 5. Results of the high-power rf testing of $644 \mathrm{MHz} \beta_{\mathrm{opt}}=$ 0.65 cavities at MSU/FRIB. $2 \mathrm{~K} Q$ curves of the BCP $+120^{\circ} \mathrm{C}$ baking treatment (green) compared to the previous $\mathrm{EP}+120^{\circ} \mathrm{C}$ baking treatment (red). The maximum gradient was limited in the $\mathrm{BCP}+$ baking test by field emission. The open circles depict field-emission $\mathrm{x}$ rays, read on the right-hand vertical scale.

$B_{\mathrm{pk}}=62 \mathrm{mT}$ is valid to compare against other rf surface treatments.

Despite the generally inferior performance we observed, $120^{\circ} \mathrm{C}$ baking can still impart some benefits that do not manifest in the Q-curves, namely a reduction in multipacting (MP) strength. However, in the EP-only case, MP behavior was minimal and strong or hard MP barriers in the MP band were not encountered. The measured MP band for this cavity is centered at $E_{\mathrm{acc}}=10 \mathrm{MV} / \mathrm{m}$ or $B_{\mathrm{pk}}=44 \mathrm{mT}$, which corresponds to first-order two-point MP at the cell equators. The first-order two-point MP resonance condition on a 'flat-like' surface is

$$
\omega_{R}=\frac{e B_{\mathrm{pk}}}{m_{e}}=2 \omega_{\mathrm{rf}}
$$

where $\omega_{R}$ is the angular electron cyclotron resonance frequency at a given $B_{\mathrm{pk}}, e$, and $m_{e}$ are the charge and mass of electron, $\omega_{\mathrm{rf}}$ is the angular rf frequency [31]. At $644 \mathrm{MHz}$, this resonance condition occurs at $B_{\mathrm{pk}}=46 \mathrm{mT}$, which is roughly consistent with the MP band we measured at $B_{\mathrm{pk}}=44 \mathrm{mT}$. Further, this field-strength associated with this equator MP band at $644 \mathrm{MHz}$ is similar to the fieldstrength associated with the equator MP band in $1.3 \mathrm{GHz}$ TESLA cavities, if scaled to that frequency [34]. Fig. 6 shows an example of the $644 \mathrm{MHz}$ MP band conditioning period. With gradual increases of the forward rf power, MP 


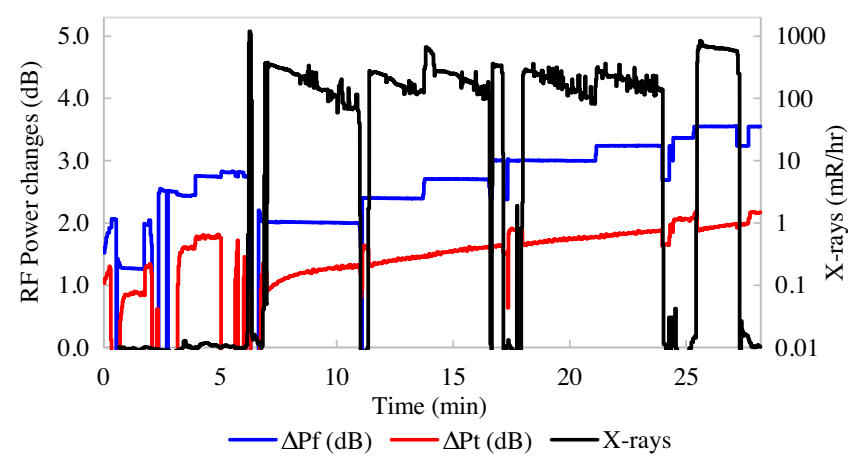

FIG. 6. RF conditioning of the cavity multipacting in the EPonly test. Pf and Pt are the forward and transmitted rf powers, respectively. The MP started to appear at an $E_{\text {acc }}$ of $9.3 \mathrm{MV} / \mathrm{m}$ $(t=0.3 \mathrm{~min}$ in this plot). The first three sudden drops of $\mathrm{Pt}(t<5 \mathrm{~min})$ are due to the MP-induced thermal breakdown with no X-rays. After these events, X-rays were detected and we performed $\mathrm{rf}$ conditioning increasing the Pf stepwise. The MP was thoroughly conditioned and disappeared at an $E_{\text {acc }}$ of $10.4 \mathrm{MV} / \mathrm{m}(t=28 \mathrm{~min})$. The X-ray detector was located to the side of the vertical test dewar, within $1 \mathrm{~m}$ from the cavity.

was successfully conditioned within $30 \mathrm{~min}$. MP strength in principle depends not only on cavity geometry but also on the cleanliness of the cavity's inner surface and the cavity vacuum quality. Since this moderate MP band was overcome with standard FRIB surface cleaning procedures and vacuum systems, we do not expect MP will be a significant issue in this style of cavity.

\section{MECHANICAL MODE STUDIES}

Anticipating cryomodule integration, the FRIB upgrade cavity's mechanical modes are important to consider, particularly in FRIB's continuous-wave cryomodules, which generally have relatively high loaded $\mathrm{Q}\left(Q_{L}\right)$ compared to pulsed cryomodules. Mechanical modes sensitive to the cavity's operating frequency of $644 \mathrm{MHz}$ can potentially drive resonant microphonics and ponderomotive instabilities [35,36,37,38]. The design of the FRIB upgrade cavity includes stiffening rings to reduce mechanical vibrations within the cavity and reduce its displacement sensitivity to the rf resonant frequency. The steep sidewalls of the FRIB upgrade cavity make it particularly susceptible to an "accordion" or longitudinal mode compared to the TESLA cavity shape, whose sidewalls are much less steep with a more rigid cell.

The mechanical simulations predicted the mechanical resonances including the transverse cavity modes at 72 and $91 \mathrm{~Hz}$, the cavity-bellows accordion mode at $94 \mathrm{~Hz}$, the cavity twist mode at $123 \mathrm{~Hz}$ [2]. The cavity-bellows accordion mode is expected to be the dominant mechanical mode, strongly coupled to the rf frequency. Usually, cavity mechanical modes are characterized in cold tests with helium-jacketed cavities equipped with tuners and with means to measure the Lorentz transfer function or piezo transfer function. However, as we were interested in the cavity mechanical mode of this new shape cavity, particularly damping of the accordion mode, the mechanical modes were measured using the available undressed fivecell cavities on a test bench at room temperature [39]. The undressed cavity was fitted with two adjustable but rigid stainless steel bars clamped to the ends of the cavity, as shown in Fig. 7, effectively pinning the ends as if a helium jacket were present. In the accordion mode, this frame provides the same constraints as the real helium jacket since the displacements are in the longitudinal direction. In the other mechanical modes such as the transverse and twist modes, their amplitudes may be exaggerated as the frame does not provide the same stiffness against bending or torsion as the real helium jacket; however, these modes are not interesting modes in this measurement since they are not strongly coupled to the rf frequency. A mechanical speaker was then fitted to the cavity test stand, and driven with a sine wave scanning from 0 to $200 \mathrm{~Hz}$. $200 \mathrm{~Hz}$ was chosen as a maximum because

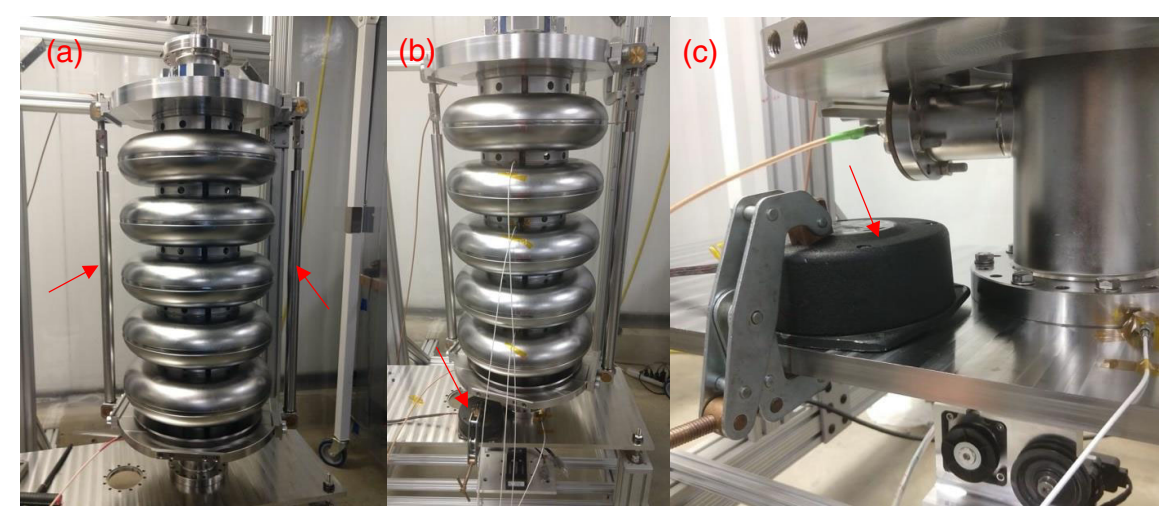

FIG. 7. (a) $644 \mathrm{MHz}$ cavity assembled with the rigid steel bars (arrows) clamped to the ends of the cavity to mimic the presence of the helium jacket. (b) Cavity assembled on the test stand with the mechanical speaker (arrow) in place, with accelerometers (white cables) attached to the cell equators. (c) details of the mechanical speaker coupled to the cavity test stand. 
possible external microphonics disturbances other than the cavity mechanical modes are within the range up to several tens of $\mathrm{Hz}$ based on experience with the FRIB cryomodules. Furthermore, simulations predict the FRIB upgrade cavity "accordion mode" is at $95 \mathrm{~Hz}[2,40]$.

While the cavity was being mechanically scanned, the cavity accelerating rf mode was excited and the sidebands were measured on a real-time spectrum analyzer, which provides $0.1 \mathrm{~Hz}$ minimum resolution bandwidth. As the scan progressed, the dominant resonance coupled to the rf mode appeared at $96 \mathrm{~Hz}$ as shown in Fig. 8, consistent with predictions. Accelerometers attached to the cavity cells show sinusoidal oscillations with a phase advance per cell as shown in Fig. 9, roughly consistent with the predicted accordion mode $[2,41]$. We also measured the decay time of

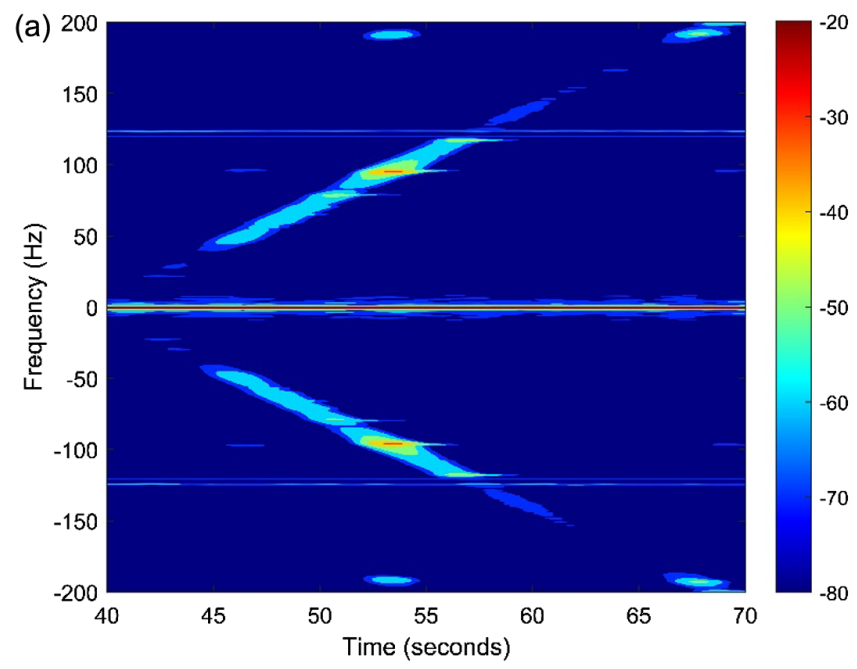

(b)

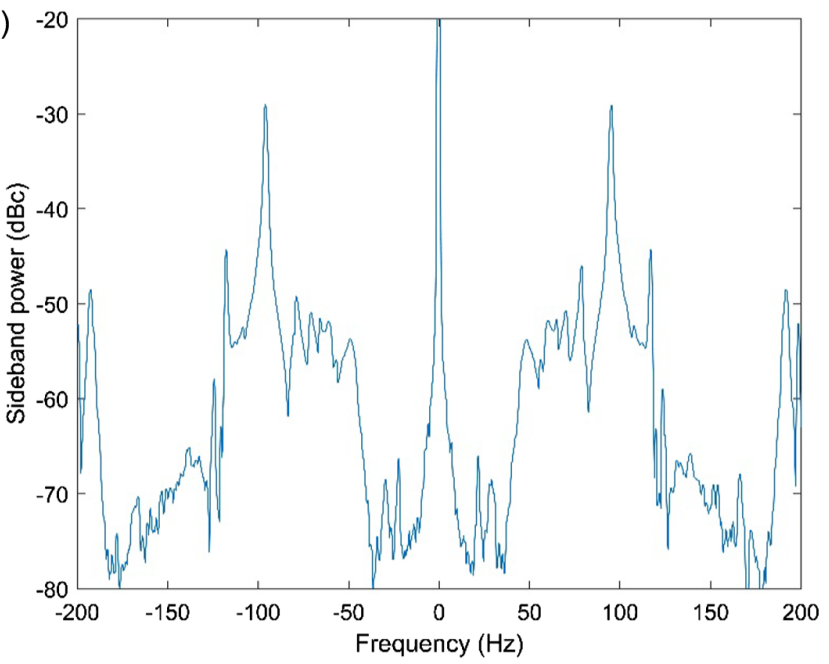

FIG. 8. (a) The spectrogram with the mechanical mode scan. Sinusoidal vibrations were excited and its frequency was swept from 20 to $200 \mathrm{~Hz}$. The color map represents the sideband power in units of $\mathrm{dBc}$. (b) The integrated spectrum. The dominant electromechanical resonance is at $96 \mathrm{~Hz}$, with a response at least $15 \mathrm{~dB}$ stronger than any other mode.

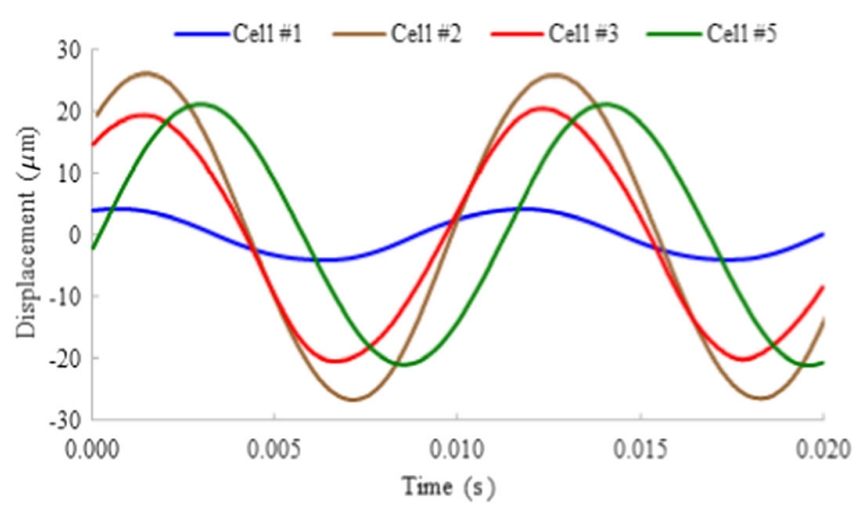

FIG. 9. Displacement oscillations at the $95 \mathrm{~Hz}$ mechanical mode. These are measured from the accelerometers attached on the equators and aligned to the cavity axis (longitudinal direction). The phase advances are $28^{\circ}$ from Cell \#1 to Cell \#2, $-4^{\circ}$ from Cell \#2 to Cell \#3, $52^{\circ}$ from Cell \#3 to Cell \#5.

this sideband to characterize the mechanical mode $\mathrm{Q}$ factor. The measured decay time is $-14 \mathrm{~dB} / \mathrm{sec}$ as shown in Fig. 10 which yields the mechanical Q of 185. This mechanical mode $\mathrm{Q}$ is no higher than that in $1.3 \mathrm{GHz}$ TESLA cavities or FRIB $\beta_{\text {opt }}=0.53322 \mathrm{MHz}$ half-wave resonators $[40,41]$.

The relationship between the rf sideband decay time and the mechanical mode $\mathrm{Q}$ was derived as follows: when the accordion mode is excited with the displacement amplitude $\Delta x$, the rf resonant frequency oscillates with the frequency excursion amplitude $\Delta f$, which results in the phase modulation with amplitude $\Delta \phi$ in the rf circuit. The mechanical displacement is much smaller than the cavity size, thus $\Delta f \propto \Delta x$. The frequency excursion is much smaller than the room temperature cavity rf bandwidth, thus $\Delta \phi \propto \Delta f$ and $\Delta \phi \ll 1$. The first sideband "power" is proportional to $J_{1}^{2}(\Delta \phi)$, where $J_{1}(x)$ is the first-order Bessel function with argument $x$. Since $\Delta \phi \ll 1$, the sideband power is approximately proportional to $\Delta \phi^{2}$ and thus proportional to $\Delta x^{2}$. Therefore, the decay time of the rf sideband power is the same as the decay time of the energy of the mechanical oscillation and the mechanical mode Q factor $Q_{m}$ can be represented by

$$
Q_{m}=\omega_{m} \tau_{\mathrm{SB}}
$$

where $\omega_{m}$ is the modulation frequency and $\tau_{\mathrm{SB}}$ is the exponential decay time of the first rf sideband power.

\section{DISCUSSION}

We find herein that the FRIB $644 \mathrm{MHz} \beta_{\mathrm{opt}}=0.65$ fivecell elliptical cavity meets and exceeds FRIB upgrade goals for $Q_{0}$ and $E_{\text {acc }}$ with an EP-only treatment, and thus is a suitable candidate cavity design in which to develop future high-Q treatments such as N-doping for the FRIB400 upgrade. With a cavity $Q_{0}$ of $2.3 \times 10^{10}$ at $17.5 \mathrm{MV} / \mathrm{m}$ 

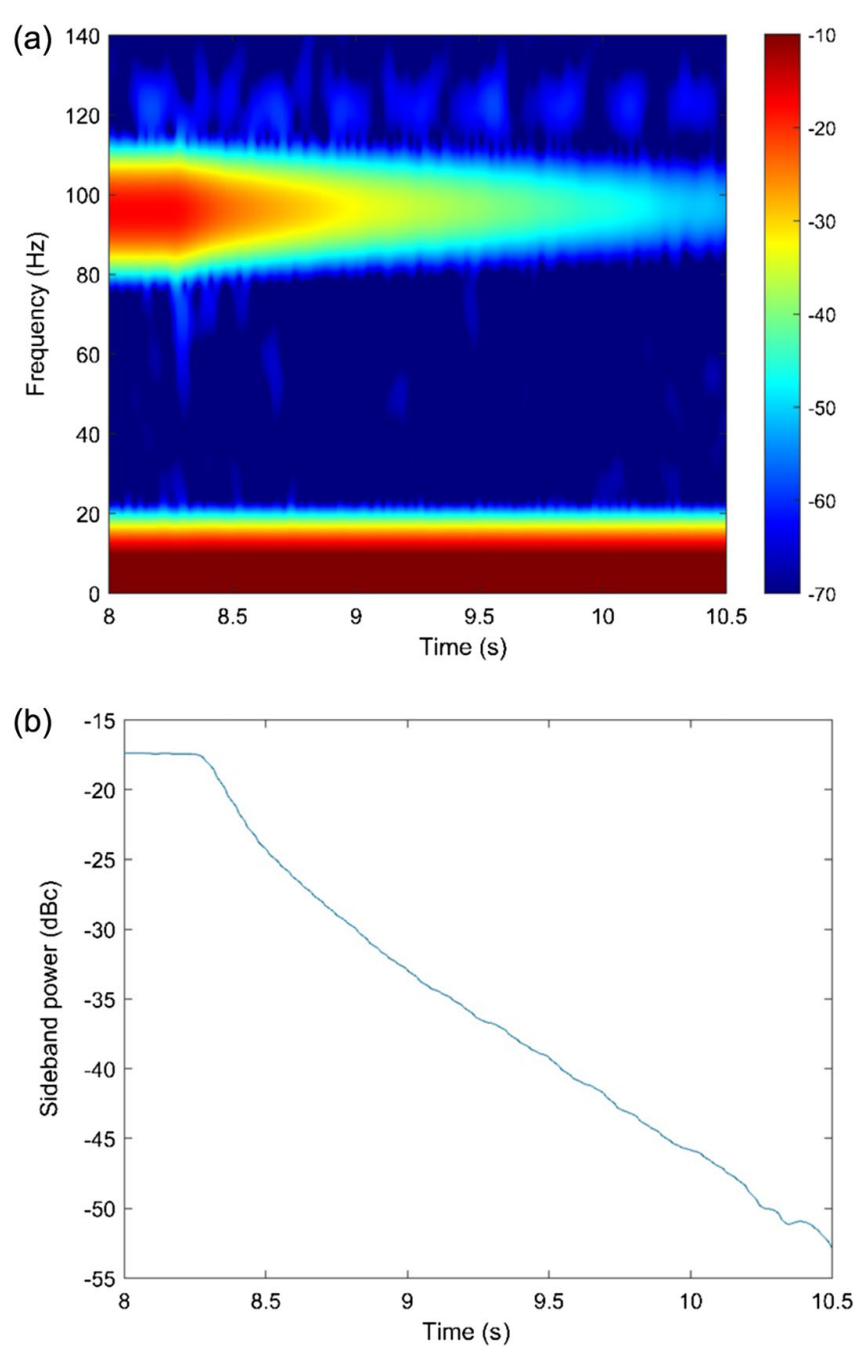

FIG. 10. (a) Spectrogram of the decay time measurement of the dominant $95 \mathrm{~Hz}$ mechanical sideband mode. This mode was excited with the mechanical speaker, which was shut off at $t=8.25 \mathrm{~s}$. The color map represents the sideband power in the unit of dBc. (b) The decay curve measured at $95 \mathrm{~Hz}$. The decay rate of the mechanical mode energy is $-14 \mathrm{~dB} / \mathrm{s}$.

and an R/Q of $368 \Omega$, we calculate the dynamic heat load for a single cavity to be $18.2 \mathrm{~W}$, falling within the design budget of $20 \mathrm{~W}$ [2].

We found that $120^{\circ} \mathrm{C}$ baking did not improve the cavity performance at $17.5 \mathrm{MV} / \mathrm{m}$ of either the $\mathrm{BCP}$ or $\mathrm{EP}$ treatments. Since $17.5 \mathrm{MV} / \mathrm{m}$ resides well within the region of the Q-curve dominated by the medium-field Q-slope, $120^{\circ} \mathrm{C}$ baking would have had to reduce the MFQS to improve cavity performance at this operating gradient. However, as in both theoretical and experimental studies $[26,28,32]$ it has been established that $120^{\circ} \mathrm{C}$ baking increases the MFQS, and our results confirm these findings, which, in theory, would not motivate further pursuit of this treatment for high- $\mathrm{Q}_{0}$ at $17.5 \mathrm{MV} / \mathrm{m}$ if this had been of interest.
While $120^{\circ} \mathrm{C}$ baking is known to reduce $R_{\mathrm{BCS}}$, we show that it increases the cavity $R_{0}$, which is the dominant contributor to $R_{\mathrm{S}}$ at the FRIB cavity operating temperature of $2 \mathrm{~K}$. With the FRIB cavity operating at $644 \mathrm{MHz}$ and $B_{\mathrm{pk}}$ of $77.5 \mathrm{mT}$, it is significant that the portion of the residual resistance due to the trapped magnetic flux $\left(R_{\mathrm{H}}\right)$ scales with the square root of the operating frequency, whereas $R_{\mathrm{BCS}}$ scales with the square of the operating frequency. Thus, higher-frequency cavities suffer more from $R_{\mathrm{BCS}}$ and suffer less from $R_{\mathrm{H}}$ contributions to $R_{0}$. Higher frequency cavities thus benefit more by $120^{\circ} \mathrm{C}$ baking than the $644 \mathrm{MHz}$ cavity tested in this work.

$120^{\circ} \mathrm{C}$ baking is also known to mitigate the high-field Q-slope. However, in a conservative estimation, the FRIB $644 \mathrm{MHz}$ cavity would not see improvements in Q from mitigation of the high-field Q-slope until the cavity operating gradient increases beyond at least $26 \mathrm{MV} / \mathrm{m}$, which is not an operational possibility.

Regarding the stability of the resonance control, the lowfrequency accordion mode of the undressed cavity was measured and found to be weakly powered and of a smaller Q than other mechanical modes observed in the other cavities currently installed and operating in the FRIB linac. This is a promising result as the dominant mechanical mode will not be resonantly built up by external vibrations (microphonics) or interaction with the Lorentz force detuning effect (ponderomotive instability). The next step in the resonance control study is characterizing the dynamic Lorentz force detuning effect in helium jacketed cavities. The final goal is validating the stability of the resonance control in a cryomodule fully integrated with the low-level rf control and cryogenics systems.

Readers may note that several cold tests revealed early onset of field emission: only one test achieved high gradient $(26 \mathrm{MV} / \mathrm{m})$ without it. Detailed analysis of the rf surface processing and clean assembly procedures at MSU revealed that the existing robotic HPR system, developed for the FRIB half-wave resonators (HWRs), was sub-optimal for the large elliptical cavity shape, and was the most likely cause of the field emission. The main drawback of the HPR system was the limited sweep angle of the rinsing wand which resulted in using a relatively large number of nozzles (18) with lower jet speeds. The reduction from 18 to 12hole nozzles and improved programming of the wand motion through the cavity pushed the onset of field emission from mid- $E_{\text {acc }}$ range upward to $25 \mathrm{MV} / \mathrm{m}$. Currently, this HPR system is being modified for better accommodation of the multicell low-frequency elliptical cavities. A rotational HPR system enables continuous rotation of the cavity, which in turn allows the number of water jet nozzles to be reduced to only 2 or 3 , increasing the jet velocity and improving other parameters such that uniform coverage of all rf surfaces can be achieved. In HPR processing at ANL in collaboration with FNAL and ANL, in preliminary investigation of nitrogen doping, no field 
emission was observed in these cavities after surface processing in these facilities. This demonstrates that despite the larger cavity diameters and the steeply sloping sidewalls, it is possible to process them to a field emissionfree state.

Since the conclusion of this study, further EP data have been collected in field-emission free trials, and no significant change is observed in the rf performance, proving the FE reported herein was not the cause of lower Q in EP-only cavity trial.

Even though the EP with the current cathode achieved remarkable cavity performance, the uniformity of surface removal with the EP is not fully optimized yet. As it is claimed that the performance of the nitrogen-doped cavities is sensitive to the thickness of the post EP, further study on the uniformity of surface removal and improvements in the cathode design could be important for future high-Q R\&D with advanced surface treatments such as nitrogen doping.

At this point, additional effort will be needed to repeat the performance of the undressed cavity in a jacketed cavity, and then in turn, in an FRIB Energy Upgrade cryomodule. In the course of this, more development and integrated testing of the cavity and cryomodule subsystems will be needed. These include fast/slow piezo and mechanical tuner studies, with the goal of improving the dynamic range and operational lifetimes of these devices before application in an FRIB energy upgrade cryomodule $[2,42,43]$. Ultimately, full integration and testing with the cryogenics system and cryomodule assembly will be required to establish how well the integrated cavity performance compares to the undressed cavity performance described here.

Despite meeting the minimum upgrade requirements in terms of $Q_{0}$ and $E_{\text {acc }}$, there is strong motivation to proceed with advanced surface treatment studies for these cavities, in particular, with $\mathrm{N}$-doping. $\mathrm{N}$-doping in other frequency and velocity regimes has proven a strong candidate for increasing cavity $Q_{0}$, especially in $1.3 \mathrm{GHz}$ TESLA cavities $[5,6,7]$, however, more work is needed to understand how to adapt successful $\mathrm{N}$-doping techniques to different frequency and velocity regimes. Initial studies of $\mathrm{N}$-doping as a function of cavity frequency have found that $\mathrm{N}$-doping in the $650 \mathrm{MHz}$ range using a " $2 / 6$ " recipe ( 2 min nitrogen infusion, 6 min annealing at high temperature) increased the cavity's BCS resistance as a function of field, whereas for $1.3,2.6$, and $3.9 \mathrm{GHz}$, BCS resistance decreased as a function of field, with the size of the drop increasing with the operating frequency [43]. This amounts to a removal of the classic "anti-Q slope" associated with $\mathrm{N}$-doping, which is detrimental as this anti-Q slope is necessary to achieve the multiple-factors of $\mathrm{Q}$ improvement we seek in this cavity and with this treatment. However, the mechanism of $\mathrm{N}$-doping is not well understood as a function of frequency, and development is needed to understand how to optimize $\mathrm{N}$-doping recipes and post $\mathrm{N}$-doping EP treatment for the
FRIB Energy Upgrade cavity in this frequency and velocity range. We therefore regard the prototype cavities as an additional opportunity to study the fundamental physics of the frequency dependence of the $\mathrm{N}$-doping technique $[43,44]$. An understanding of this dependence and how to manipulate it will be broadly useful in designing $\mathrm{N}$-doping recipes for a range of cavities for a range of applications where high-Q will be useful in experimental physics as well as in industry.

\section{CONCLUSIONS}

At the conclusion of this study, we find that the novel FRIB400 prototype $644 \mathrm{MHz}$ five-cell elliptical medium- $\beta$ cavity achieves acceptable performance with conventional rf surface processing techniques, proving that the cavity and its novel geometry are sound candidates for future high-Q rf surface processing development. The cavity is currently capable of meeting the design goal of the FRIB energy upgrade with EP-only surface treatment. At the FRIB operating gradient of $17.5 \mathrm{MV} / \mathrm{m}$, the EP-only trial gave a higher $\mathrm{Q}_{0}$ than the $\mathrm{EP}+$ baking recipe due to lower residual resistance and a lower medium-field Q slope. Concerns about MP and mechanical modes introduced by a new cavity shape were alleviated based on the results we present here. MP conditioning was completed in the continuous-wave mode and did not require additional pulsed conditioning. The cavity's accordion mechanical mode Q is somewhat similar to that of the TESLA cavity and it is lower than that in the existing FRIB low- $\beta$ cavities, which is a promising result regarding the stability of the resonance control since the dominant mechanical mode will not be resonantly built up by microphonics or ponderomotive instability.

\section{ACKNOWLEDGMENTS}

This work is supported by the Michigan State University and US Department of Energy, Office of Science, High Energy Physics Award No. DE-SC0020371. This work is additionally supported by the US Department of Energy, Office of Science, High Energy Physics under Cooperative Agreement Award No. DE-SC0018362.

[1] FRIB400 White Paper, https://indico.frib.msu.edu/event/2/ attachments/57/292/FRIB400_Final.pdf.

[2] P. N. Ostroumov, C. Contreras, A. S. Plastun, J. Rathke, T. Schultheiss, A. Taylor et al., Elliptical superconducting RF Cavities for FRIB energy upgrade, Nucl. Instrum. Methods Phys. Res., Sect. A 888, 53 (2018).

[3] B. Aune et al., Superconducting TESLA cavities, Phys. Rev. ST Accel. Beams 3, 092001 (2000).

[4] K. Saito et al., Superiority of electropolishing over chemical polishing on high gradients, in Contribution to 8th 
Workshop on RF Superconductivity [Part. Accel. 60, 193 (1998)], https://inspirehep.net/literature/470403.

[5] S. Michizono, The International Linear Collider, Nat. Rev. Phys. 1, 244 (2019), https://doi.org/10.1038/s42254-0190044-4.

[6] W. Singer et al., Production of superconducting $1.3 \mathrm{GHz}$ cavities for the European x-ray free electron laser, Phys. Rev. Accel. Beams 19, 092001 (2016).

[7] A. Grassellino, A. Romanenko, D. Sergatskov, O. Melnychuk, Y. Trenikhina, A. Crawford, A. Rowe, M. Wong, T. Khabiboulline, and F. Barkov, Nitrogen and argon doping of niobium for superconducting radio frequency cavities: A pathway to highly efficient accelerating structures, Supercond. Sci. Technol. 26, 102001 (2013).

[8] A. Grassellino et al., Unprecedented quality factors at accelerating gradients up to $45 \mathrm{MV} / \mathrm{m}$ in niobium superconducting resonators via low temperature nitrogen infusion, Supercond. Sci. Technol. 30, 094004 (2017).

[9] D. Gonnella, R. Eichhorn, F. Futura, M. Ge, D. Hall, V. Ho et al., Nitrogen-doped 9-cell cavity performance in a test cryomodule for LCLS-II, J. Appl. Phys. 117, 023908 (2015).

[10] D. Gonnella, S. Aderhold, A. Burrill, E. Daly, K. Davis, A. Grassellino et al., Industrialization of the nitrogen-doping preparation for SRF cavities for LCLS-II, Nucl. Instrum. Methods Phys. Res., Sect. A 883, 143 (2018).

[11] Z. A. Conway, A. Barcikowski, G. L. Cherry, R. L. Fischer, S. M. Gerbick, C. S. Hopper et al., Achieving high peak fields and low residual resistance in half-wave cavities, in Proceedings of SRF 2015 (JACoW, Geneva, 2015), p. 973, https://accelconf.web.cern.ch/SRF2015/.

[12] T. Xu, K. Saito, H. Ao, B. Bird, N. K. Bultman, F. Casagrande et al., Progress of FRIB SRF production, in Proceedings of SRF2017 (JACoW, Geneva, 2019), p. 345, https://accelconf.web.cern.ch/napac2019/.

[13] W. Hartung et al., Large-scale dewar testing of FRIB production cavities: Results, in Proceedings of NAPAC 2019 (Lansing, MI, USA, 2019), MOPLO17.

[14] L. Lilje, High gradients in superconducting multi-cell cavities, in Proceedings of 11th Workshop on RF Superconductivity (SRF'03) (JACoW, Geneva, 2003), p. 220, https://accelconf.web.cern.ch/SRF2003/INDEX.htm.

[15] M. Drury, E. Daly, G. K. Davies, J. R. Delayen, C. Grenoble, R. Hicks et al., Overview of SNS Cryomodule Performance, in Proceedings of 2005 Particle Accelerator Conference (Knoxville, Tennessee, 2005).

[16] A. Bosotti, A. Bellandi, M. Bertucci, A. Bignami, J. F. Chen, C. G. Maiano et al., Vertical Tests of ESS Medium $\beta$ Prototype Cavities at LASA., Proceedings of IPAC17 (Copenhagen, Denmark, 2017), MOPVA063.

[17] M. Kelly and T. Reid, Surface processing for bulk niobium superconducting radio frequency cavities, Supercond. Sci. Technol. 30, 043001 (2017).

[18] C. Z. Antoine, A. Aspart, M. Berthelot, Y. Gasser, J. P. Poupeau, and V. Valin, Morphological and Chemical Studies of Nb Samples After Various Surface Treatments, in Proceedings of SRF99 (Santa Fe, New Mexico, 1999), p. 295, TUP035.

[19] M. Bertucci, A. Bosotti, A. D’Ambros, P. Michelato, L. Monaco, C. Pagani et al., Electropolishing of PIP-II low $\beta$ cavity prototypes, in Proceedings of SRF2019 (Dresden, Germany, 2019), MOP057.

[20] G. Ciovati, G. Myneni, F. Stevie, P. Maheshwari, and D. Griffis, High field Q slope and the baking effect: Review of recent experimental results and new data on $\mathrm{Nb}$ heat treatments, Phys. Rev. Accel. Beams 13, 022002 (2010).

[21] J. Mammosser, J. Delayen, J. Gordon, L. Phillips, A. M. Valente, T. Wang, A. T. Wu, and J. Saunders, Status of the production electropolishing system at JLab, in Proceedings of 2003 Particle Accelerator Conference (2003), p. 2860.

[22] M. S. Champion, R. A. Dean, J. D. Galambos, M. P. Howell, M. A. Plum, and B. W. Riemer, Progress on the proton power upgrade of the spallation neutron source, in Proceedings of IPAC2017 (2017), p. 2445.

[23] L. Merminga, Building for Discovery: The PIP-II Project (ORNL Virtual seminar, 2021).

[24] H. Padamsee, RF Superconductivity: Science, Technology, and Applications, (WILEY-VCH Verlag GmbH \& Co. KGaA, Weinheim, 2009), ISBN: 978-3-527-40572-5.

[25] D. Dotson, M. Drury, R. May, and C. Reece, Use of simple $\mathrm{X}$-ray measurements in the performance analysis of cryogenic RF accelerator cavities, Report No. Jlab-ACC-96-11, 1996, https://www.jlab.org/div_dept/admin/publications/ papers/96/ACC96-11.pdf

[26] G. Ciovati and J. Halbritter, Analysis of the medium field Q-slope in superconducting cavities made of bulk niobium, Physica (Amsterdam) 441C, 57 (2006).

[27] J. Halbritter, Transport in superconducting niobium films for radio frequency applications, J Appl. Phys. 97, 083904 (2005).

[28] G. Ciovati, High Q at Low, and Medium Field, in Proceedings of Argonne Frontier Workshop (2005), edited by K. Kim et al. (JLab MIS portal, 2005), p. 52, https:// misportal.jlab.org/ul/publications/downloadFile.cfm? hyperlink=ACF2B2C.pdf.

[29] G. Ciovati, P. Kneisel, and A. Gurevich, Measurement of the high-field Q drop in a high-purity large-grain niobium cavity for different oxidation processes, Phys. Rev. Accel. Beams 10, 062002 (2007).

[30] P. Dhakal, G. Ciovati, and G. Myneni, Role of thermal resistance on the performance of superconducting radio frequency cavities, Phys. Rev. Accel. Beams 20, 032003 (2017).

[31] H. Padamsee, RF Superconductivity for Accelerators (WILEY-VCH Verlag GmbH \& Co. KGaA, Weinheim, 2008), ISBN: 978-3-527-40842-9.

[32] J. Vines, Y. Xie, and H. Padamsee, Systematic trends for the medium field $\mathrm{Q}$ slope, in Proceedings of the 13th International Conference RF Superconductivity (SRF'07) (Beijing, China 2007), TUP27.

[33] L. Lilje, D. Reschke, K. Twarowski, P. Schmüser, D. Ploess, E. Haebel et al., Electropolishing and in-situ baking of $1.3 \mathrm{GHz}$ niobium cavities, in Proceedings of 1999 Workshop on RF Superconductivity (Santa Fe, New Mexico, USA).

[34] P. Yla-Oijala, Electron multipacting in TESLA cavities and input couplers, Part. Accel. 63, 105 (1999), https:// inspirehep.net/literature/514939.

[35] J. Delayen, Ponderomotive instabilities and microphonicsa tutorial, Physica (Amsterdam) 441C, 1 (2006). 
[36] S. Kim, RF commissioning of the first three Beta $=0.041$ quarter wave resonator cryomodule in FRIB driver linac, in Second Topical Workshop on Microphonics (New York, NY, 2018).

[37] L. Yang, V. Zvyagintsev, R. E. Laxdal, and B. Waraich, Damping of vibrations in superconducting quarter wave resonators, Phys. Rev. Accel. Beams 22, 030103 (2019).

[38] Y. Pichalnikov, FNAL tuners: LCLS-II to PIP-II, Second Topical Workshop on Cryomodule Microphonics and Resonance Control (New York, NY, 2018).

[39] S. Kim, Resonance control with pneumatic slow frequency tuners for FRIB half-wave resonators, TESLA Technology Collaboration Meeting 2020 (Geneva, Switzerland, 2020).

[40] L. Lilje, S. Simrock, and D. Kostin, Characteristics of a fast piezo-tuning mechanism for superconducting cavities, in Proceedings of EPAC 2002 (EPAC, 2002), p. 2256, https:// accelconf.web.cern.ch/e02/default.htm.
[41] O. Kononenko, C. Adolphsen, Z. Li, C.-K. Ng, and C. Rivetta, 3D multiphysics modeling of superconducting cavities with a massively parallel simulation suite, Phys. Rev. Accel. Beams 20, 102001 (2017).

[42] M. Martinello, S. Aderhold, S. K. Chandrasekaran, M. Checchin, A. Grassellino, O. Melnychuk et al., Advancement in understanding of the field and frequency dependent microwave surface resistance of Niobium, in Proceedings of SRF 2017 (JACoW, Geneva, 2017), arXiv:1707.07582, https://accelconf.web.cern.ch/srf2017/.

[43] M. Checchin, M. Martinello, A. Grassellino, S. Aderhold, S. K. Chandrasekaran, O. S. Melnychuk, S. Posen, A. Romanenko, and D. A. Sergatskov, Frequency dependence of trapped flux sensitivity in SRF cavities, Appl. Phys. Lett. 112, 072601 (2018).

[44] M. P. Kelly, S. M. Gerbick, D. Bice, and G. Wu, Electropolishing at ANL/FNAL, in Proceedings of SRF2009 (Berlin, Germany) (2009), THPPO066. 